Informe especial

\title{
Adaptación cultural de instrumentos utilizados en salud ocupacional
}

\author{
Neusa Maria Costa Alexandre ${ }^{1}$ y Edinêis de Brito Guirardello ${ }^{1}$
}

RESUMEN Se han creado numerosos instrumentos estandarizados para evaluar el estado de salud y la eficacia de los tratamientos. Sin embargo, la mayoría de ellos han sido desarrollados y validados en la lengua inglesa. En la literatura se recomienda la utilización de escalas y cuestionarios estandarizados cuyos resultados puedan ser comparados internacionalmente. Las diferencias culturales no permiten hacer una simple traducción de estos instrumentos. En este artículo se resumen normas sistematizadas para la adaptación cultural de instrumentos utilizados en salud ocupacional, derivadas de investigaciones especializadas.

Palabras clave Adaptación cultural, traducción, salud ocupacional.

Existen importantes cuestionarios y escalas muy utilizados en salud ocupacional para evaluar el estado de salud, la capacidad funcional, la localización e intensidad del dolor, la calidad de vida y los aspectos psicosociales de pacientes con diversas enfermedades (1-5). Esos instrumentos se han utilizado en investigaciones en la práctica clínica para evaluar la eficacia de tratamientos o intervenciones, en la evaluación de los servicios de salud y en programas de salud pública. Sin embargo, la mayoría de ellos han sido desarrollados y validados en la lengua inglesa. En la literatura se recomienda adaptar escalas y cuestionarios ya validados, argumentando que eso reduce los costos y faci-

\footnotetext{
1 Departamento de Enfermería, Facultad de Ciencias Médicas, Universidad Estatal de Campinas, São Paulo, Brasil. Toda la correspondencia debe ser enviada a Neusa M. C. Alexandre a la siguiente dirección: Departamento de Enfermagem, Faculdade de Ciências Médicas, Universidade Estadual de Campinas (UNICAMP), Cidade Universitária "Zeferino Vaz", CEP: 13083.300 Campinas - São Paulo — Brasil. Correo electrónico: neusalex@fcm. unicamp.br
}

lita el intercambio de información entre la comunidad científica $(6,7)$.

La adaptación de un instrumento a otro idioma es un proceso complejo. Debido a las diferencias culturales, no se puede realizar una simple traducción. Según Guillemin (6), en el proceso de adaptación a otra lengua se deben considerar de forma distinta la cultura y el país implicados, teniendo en cuenta el idioma, el contexto cultural y el estilo de vida. Este punto debe ser considerado principalmente en las poblaciones hispánicas que viven en otros países, como los Estados Unidos, y que, aun teniendo el mismo idioma, poseen dialectos propios que necesitan ser tomados en consideración (8). Dentro de un nuevo contexto de las ciencias humanas y biológicas, Patrick et al. (9) reflexionan que es esencial entender en qué medida los valores relacionados con el estado de salud son similares en diferentes culturas y grupos sociales. Así, para adaptar un instrumento a otro idioma deben tenerse en cuenta aspectos técnicos, lingüísticos y semánticos (10). Para asegurar la adaptación cultural de los instrumentos de una forma metodológicamente correcta, en la literatura internacional se han propuesto normas sistematizadas que resumimos en este artículo.

\section{PROCEDIMIENTO METODOLÓGICO DE ADAPTACIÓN CULTURAL}

Numerosos investigadores han realizado adaptaciones de complejos e importantes instrumentos (11-15). Muchos países han desarrollado o adaptado escalas y cuestionarios a través de proyectos cooperativos multicéntricos, utilizando protocolos metodológicos sistematizados (15-16). La tendencia actual es estandarizar normas internacionales para asegurar la calidad de los instrumentos adaptados. En el presente estudio se resumen los pasos esenciales que debe incluir el proceso de adaptación, derivados principalmente de publicaciones especializadas $(6,7,17)$. 


\section{Traducción inicial}

La traducción inicial debe ser hecha al menos por dos traductores independientes que sean profesionales calificados y que tengan como lengua materna el idioma de destino (17). Esto permite la detección de errores y la interpretación divergente de ítems con significados ambiguos en el instrumento original. La mitad de esos traductores debe conocer los objetivos del instrumento y los conceptos implicados, mientras que la otra mitad debe hacer la traducción sin ese conocimiento (18). El conocimiento de los objetivos permitirá una equivalencia cultural e idiomática, y no solo una equivalencia entre las dos versiones, con lo cual se obtendrá un instrumento más confiable. Por otro lado, el desconocimiento de los objetivos será útil para extraer significados inesperados del instrumento original. Este procedimiento facilitará la traducción conceptual y la literaria simultáneamente.

\section{Retrotraducción}

La traducción inicial debe ser vertida nuevamente al idioma original por otros traductores que no hayan participado en la primera etapa y que trabajen de forma independiente $(6,18)$. Esos traductores deben ser bilingües, tener como lengua materna el idioma en el que se escribió originalmente el instrumento y desconocer sus fines $\mathrm{y}$ conceptos, disminuyendo así la posibilidad de sesgos y expectativas (17).

\section{Revisión por un comité}

Un comité multidisciplinario debe revisar y comparar las traducciones realizadas hasta la obtención de la versión final (7), además de revisar las instrucciones para la cumplimentación del instrumento y su puntuación. El comité debe estar compuesto por personas bilingües y especialistas en el área de conocimiento del instrumento, que necesitan ser informadas sobre las medidas y conceptos implicados (17). Algunos autores también recomiendan la inclusión de personas legas $(6,10)$. Cuando se trate de una adaptación cultural a una población inmigrante, se recomienda la participación de personas representativas de esa población.

Ese comité debe cerciorarse de que la versión final sea totalmente comprensible y evaluar su equivalencia cultural. Es necesario que exista una equivalencia semántica, idiomática, conceptual y experimental (6). Dentro de este contexto, el comité puede modificar las orientaciones iniciales y el formato del instrumento, alterar o rechazar ítems inadecuados e incluso crear nuevos ítems. Para comprobar que el instrumento está realmente escrito en un lenguaje comprensible para una gran parte de las personas, se recomienda que pueda ser entendido por individuos de 10 a 14 años $(7,19,20)$.

\section{Prueba preliminar}

La versión final debe ser sometida a una prueba preliminar para evaluar su equivalencia con la versión original. Esta prueba consiste en aplicar el instrumento a una muestra de población para detectar errores y confirmar que todas las preguntas pueden ser bien comprendidas. De esa forma, la prueba preliminar sirve para evaluar no solo la calidad de la traducción, sino también para verificar aspectos prácticos de su aplicación (21).

Según Guillemin et al. (17), existen dos maneras de realizar esta prueba preliminar. La primera consiste en aplicar el instrumento a un grupo de pacientes que deben ser entrevistados para identificar palabras o preguntas de difícil comprensión, para evaluar su aceptabilidad y para hacer comentarios sobre el instrumento de una forma general. Se puede registrar también el tiempo necesario para completarlo. Para hacer la equivalencia de un cuestionario sobre calidad de vida, Ciconelli et al. (14) solicitaron que 20 pacientes respondiesen a ese instrumento. En cada pregunta, los autores añadieron la opción "no aplicable", con la finalidad de identificar preguntas no comprendidas o culturalmente inadecuadas. Las preguntas cuya respuesta fue "no apli- cable" en más de un $15 \%$ de los casos fueron evaluadas nuevamente y modificadas. Al validar el cuestionario Roland-Morris, Wiesinger et al. (18) lo aplicaron a pacientes con dolor lumbar y a niños de 12 años para verificar si sería comprendido por personas de bajo nivel educacional.

La segunda forma de realizar la prueba preliminar consiste en aplicar las versiones original y final del instrumento a un grupo de personas bilingües y legas. Chang et al. (22) adoptaron este procedimiento, aplicando las versiones inglesa y china del mismo cuestionario a estudiantes bilingües del curso de graduación de enfermería. Se reformularon los ítems que fueron considerados inadecuados por más del $20 \%$ de los estudiantes.

En esta fase muchos autores han empleado un proceso muy usado en investigaciones cualitativas, que es la discusión en grupos focales con miembros de la comunidad, profesionales sanitarios y clientes $(15,23)$.

\section{Evaluación de los valores de puntuación}

Dependiendo del instrumento, puede haber necesidad de realizar una adaptación de los valores de puntuación dentro del nuevo contexto cultural. Este procedimiento puede ser hecho por un grupo compuesto por pacientes, legos y profesionales sanitarios. Otra forma puede ser mediante un abordaje matemático, utilizando procedimientos estadísticos (17).

\section{Evaluación de las propiedades psicométricas del instrumento}

Esta etapa comprende la evaluación de la validez y confiabilidad del instrumento adaptado. Sin embargo, no existe un consenso en la literatura sobre los procedimientos a seguir en esta fase de la adaptación cultural $(6,7)$. Guillemin (6) sugiere estudiar la validez de criterio y de concepto y la fiabilidad intraobservador e interobservadores. La validación permite comprobar si el instrumento mide exactamente lo que se 
propone medir (24). En la literatura se han descrito diferentes métodos para verificar la validez: validez de contenido, de criterio y de concepto, todos ellos con sus subdivisiones (25). La utilización de esos métodos depende del tipo de información y de la investigación. La fiabilidad representa la coherencia de la medida que se quiere obtener (26) y sus principales atributos (estabilidad, homogeneidad y equivalencia) pueden ser examinados a través de diferentes procedimientos y pruebas estadísticas.

\section{CONSIDERACIÓN FINAL}

La utilización de instrumentos estandarizados y validados en el área de la salud ocupacional tiene numerosas ventajas. Para eso es necesario realizar una adaptación cultural, procedimiento metodológico complejo que posibilita el intercambio de información entre la comunidad científica y la comparación de los resultados de las investigaciones en diferentes países.

\section{REFERENCIAS}

1. Melzack R. The McGill Pain Questionnaire: major properties and scoring methods. Pain 1975;1:277-299.

2. Corlett EN, Bishop RP. A technique for assessing postural discomfort. Ergonomics 1976; 19:175-182.

3. Roland M, Morris R. A study of the natural history of back pain. Part I: development of a reliable and sensitive measure of disability in low back pain. Spine 1983;8:141-144.

4. Ware JE, Sherbourne CD. The MOS 36-item Short Form Health Survey (SF 36). I. Conceptual framework and item selection. Med Care 1992;30:473-483.

5. Symonds TL, Burton AK, Tillotson KM, Main CJ. Do attitudes and beliefs influence work loss due to low back trouble? Occup Med (Lond) 1996;46:25-32.

6. Guillemin F. Cross-cultural adaptation and validation of health status measures. Scand J Rheumatol 1995;24:61-63.

7. Hutchinson A, Bentzen N, Konig-Zanhn C. Cross cultural health outcome assessment: a user's guide. Groningen: European Research Group on Health Outcomes; 1997.

8. Hendricson WD, Russell IJ, Prihoda TJ, Jacobson JM, Rogan A, Bishop GD. An approach to developing a valid Spanish language translation of a health-status questionnaire. Med Care 1989;27:959-966.

9. Patrick DL, Sittampalam Y, Somerville SM, Carter WB, Bergner M. A cross-cultural comparison of health status values. Am J Public Health 1985;75:1402-1407.

10. Hunt SM, Alonso J, Bucquet D, Niero M, Wiklund I, McKenna S. Cross-cultural adaptation of health measures. Health Policy 1991;19: 33-44.
11. Hendricson WD, Russell IJ, Prihoda TJ, Jacobson JM, Rogan A, Bishop GD, et al. Development and initial validation of a dual-language English-Spanish format for the Arthritis Impact Measurement Scales. Arthritis Rheum 1989;32:1153-1159.

12. Alonso J, Anto JM, Moreno C. Spanish version of the Nottingham Health Profile: translation and preliminary validity. Am J Public Health 1990;80:704-708.

13. González VM, Stewart A, Ritter PL, Lorig K. Translation and validation of arthritis outcome measures into Spanish. Arthritis Rheum 1995;38:1429-1446.

14. Ciconelli RM, Ferraz MB, Santos W, Meinão I, Quaresma MR. Tradução para a língua portuguesa e validação do questionário genérico de avaliação de qualidade de vida SF-36. Revista Brasileira de Reumatologia 1999;39: 143-150.

15. Fleck MPA, Leal OF, Louzada S, Xavier M, Chachamovich E, Vieira G, et al. Desenvolvimento da versão em português do instrumento de avaliação de qualidade de vida da Organização Mundial da Saúde. Revista Brasileira de Psiquiatria 1999;21:19-28.

16. Bennett K, Cardiel MH, Ferraz MB, Riedemann P, Goldsmith $\mathrm{CH}$, Tugwell P. Community screening for rheumatic disorder: cross-cultural adaptation and screening characteristics of the COPCORD Core Questionnaire in Brazil, Chile, and Mexico. J Rheumatol 1997;24:160-168.

17. Guillemin F, Bombardier C, Beaton D. Crosscultural adaptation of health-related quality of life measures: literature review and proposed guidelines. J Clin Epidemiol 1993;46: 1417-1432.
18. Wiesinger GF, Nuhr M, Quittan M, Ebenbichler G, Wölfl G, Fialka-Moser V. Cross-cultural adaptation of the Roland-Morris questionnaire for German-speaking patients with low back pain. Spine 1999;24:1099-1103.

19. Fukuhara S, Bito S, Green J, Hsiao A, Kurokawa K. Translation, adaptation, and validation of the SF-36 Health Survey for use in Japan. J Clin Epidemiol 1998;51:1037-1044.

20. Leplège A, Ecosse E, Verdier A, Perneger TV. The French SF-36 Health Survey: translation, cultural adaptation and preliminary psychometric evaluation. J Clin Epidemiol 1998;51: 1013-1023.

21. Jones EG, Kay M. Instrumentation in crosscultural research. Nurs Res 1992;41:186-188.

22. Chang AM, Chau JP, Holroyd E. Translation of questionnaires and issues of equivalence. J Adv Nurs 1999;29:316-322.

23. Knudsen HC, Vázquez-Barquero JL, Welcher B, Gaite L, Becker T, Chisholm D, et al. Translation and cross-cultural adaptation of outcome measurements for schizophrenia. Br J Psychiatry 2000;39(Supl):S8-S14.

24. LoBiondo-Wood G, Haber J. Nursing research: methods, critical appraisal, and utilization. 4th ed. St Louis: Mosby; 1998.

25. Polit DF, Hungler BP. Fundamentos de pesquisa em enfermagem. 3 ed. Porto Alegre: Artes médicas; 1995.

26. Burns N, Grove SK. The practice of nursing research: conduct, critique and utilization. 3rd ed. Philadelphia: Saunders; 1997.

Manuscrito recibido el 10 de diciembre de 2001. Aceptado para publicación el 26 de diciembre de 2001.
ABSTRACT

\section{Cultural adaptation of instruments utilized in occupational health}

Numerous standardized questionnaires and other instruments for evaluating health status and treatment effectiveness have been developed. However, the majority of these instruments have been constructed and validated in English. The scientific literature generally recommends the use of scales and standardized questionnaires whose results can be compared internationally. However, because of cultural differences, it is not sufficient to just do a simple translation of these instruments. Drawing on specialized research in this area, this article presents a summary of systematized standards for the cultural adaptation of instruments used in occupational health. 